\title{
Fusion Imaging in Nuclear Medicine-Applications of Dual-Modality Systems in Oncology
}

\author{
Orazio Schillaci and Giovanni Simonetti \\ Department of Biopathology and Diagnostic Imaging, University “Tor Vergata," Rome, Italy
}

\section{SUMMATION}

Medical imaging has become of the utmost importance in evaluating patients with cancer. Single-photon emission computed tomography (SPECT) and positron emission tomography (PET) are accurate methods for detecting cancer and related metabolic abnormalities, but they often do not provide the anatomical landmarks needed to precisely localize lesions. Magnetic resonance imaging (MRI) and computed tomography (CT) scan, on the other hand, offer excellent anatomic detail but are less sensitive because they do not provide functional detail. Fusion imaging combines functional studies with morphological ones, so overcoming the drawbacks of both modalities. Software-based fusion of independently performed scintigraphic and radiological images has proven time consuming and impractical for routine use. Recently, dual-modality integrated imaging systems (SPECT/CT and PET/CT) have been developed: the acquired images are coregistered by means of the hardware in the same session. These new devices can be particularly useful for tumour imaging. The anatomical images provide precise localization and allow the exclusion of disease in sites of physiologic tracers' accumulation for SPECT and PET findings. Hybrid imaging in oncological applications has been very encouraging, indicating that these systems are suited for routine use in clinical practice. In fact, fused images provide additional information that improves diagnostic accuracy and impacts on patient management.

Key words: fusion imaging, hybrid systems, SPECT, PET, CT

\section{INTRODUCTION}

The rapid evolution of imaging techniques has caused an increasing use of medical images in healthcare and in bio-medical research. Due to the number of imaging studies performed, it is essential to correlate one image to another to obtain the pertinent information.

Address reprint requests to: Orazio Schillaci; Viale Mazzini 121, 00195 Rome, Italy; Tel: +39-06-20902419; Fax: +39-06-37515679

E-mail: oschil@tiscali.it
In many scenarios, images from several modalities are mentally integrated by the physician to draw useful clinical conclusions, but this process is often difficult and requires mental compensation for changes in subject position. ${ }^{1}$ Computeraided techniques can be useful in this task, especially image registration that aligns the images so that features seen on different modalities can be easily related. ${ }^{2}$ In particular, intermodality registration enables complementary information from different modalities to be combined. Intramodality registration enables accurate comparisons to be 
made between images from the same modality, ${ }^{3}$ allowing monitoring of subtle changes over time. After registration, a fusion step is necessary for the integrated display of the data involved.

\section{FUSION IMAGING}

Basically, two types of medical images can be acquired: structural images that provide morphological detail, and functional images that provide physiological information. Theoretically, any type of medical image can be combined with another image to create a third image. However, the fusion of structural images (such as CT or MRI) with functional single-photon emission computed tomography (SPECT) or positron emission tomography (PET) images of the same sections of the body is especially useful. It can provide complementary anatomical and physiological information that is of great importance to diagnosis and treatment. ${ }^{4}$ Functional and structural images are now increasingly seen as complementary methods and their union can improve the data supplied by both modalities and lead to clinical information not visible in the distinct images. ${ }^{5,6}$ The ultimate purpose of image fusion is to impose a structural anatomic framework on functional images.

It is well known that nuclear medicine images demonstrate function rather than anatomy. They are very useful in the diagnosis of disease because they demonstrate changes before there are identifiable anatomical correlates and clinical manifestations. ${ }^{7}$ However, anatomic landmarks are frequently needed to precisely identify the focus of abnormal uptake and the structures containing normal activity. On the other hand, CT and MRI images provide details of normal and diseased organ anatomy. The definition of structural abnormalities is accurate, but physiologic information is not readily generated. These methods are less effective when normal anatomy is variable, as in post-surgical patients. ${ }^{8}$

Registration and fusion with a structural image can be valuable as an adjunct to interpretation of functional images, as well as offering the possibility to overcome intrinsic limitations in nuclear medicine images, like poor spatial resolution, limited signal to noise ratio, and poor tracer uptake in the diseased condition. Image fusion is useful to accurately localize tracer accumulations, to detect occult pathology, to characterize metabolically active lesions, and to draw precisely regions of interest for quantitative studies. ${ }^{9}$

A careful matching of the data from different methods is of importance for fusing two images. In the visual comparison, the images are reoriented based on the spatial sense of the diagnostician. This approach is sufficient only for a simple comparison of large lesions, with the quality and amount of information obtained degrading significantly as the size of lesions decreases. ${ }^{10}$

The use of computer-processing methods makes more exact comparison possible. ${ }^{11}$ Image registration fixes correspondence between different features visible on different imaging modalities, allows monitoring of subtle changes in size or intensity over time or across a population, and establishes correspondence between images and physical space. Establishing the correspondence of spatial information in medical images and equivalent structures in the body is fundamental to image analysis and interpretation.

The procedure of registration includes imagebased and non-image-based methods. ${ }^{10}$ Imagebased registration can be divided into extrinsic methods, based on foreign objects inserted into the imaged field, and intrinsic methods that rely only on the patient-generated image content. ${ }^{9}$

External fiducial markers attached to the body surface are employed in extrinsic methods. ${ }^{10}$ The objects attached to the patient must be visible without introducing artifacts into the images. ${ }^{9}$ It is assumed that the markers remain fixed between the different scanning sessions or that they can be accurately repositioned. A minimum of three markers are needed in each image and they are often used together with some kind of immobilizing equipment. After the acquisitions, the registration is fast and not difficult and can usually be automated without the need of complex optimization algorithms. However, external markers are less practical for routine use in clinical practice. Because of their complexity and prospective character, the markers must be placed on the patient before image acquisitions or the images cannot be registered. ${ }^{5}$

In intrinsic methods, the registration is based on a set of identified points (landmarks), on the alignment of segmented binary structures (segmentation based), or onto measures computed from the image grey values (voxel property based). ${ }^{10}$ Landmarks are anatomical $^{12}$ (at least three pairs of corresponding points in anatomical structures depicted in both images and localized 
by the user) or geometrical (corners or other geometric features generally identified in an automatic way ${ }^{13}$ ); however, the low resolution of nuclear medicine images limits their applicability.

Segmentation registration methods can be based on a rigid model where the same anatomical structures are drawn out from both images to be registered and are employed as the only input for the alignment process, or a deformable model, where an extracted structure from one image is elastically deformed into the second one. ${ }^{10} \mathrm{~A}$ popular method called "head-hat", based on surface matching of the segmented images, was introduced by Pellizzari et al. ${ }^{14}$ and applied in functional/anatomical registration. A drawback of segmentation-based methods is that the registration performance is limited to the accuracy of the segmentation step. These methods only use edge information and do not incorporate data on the internal structures of the organ. ${ }^{10}$ Edges are welldefined only on high-resolution images.

In the previously described methods, image registration is performed by identifying a small number of equivalent features like points or surfaces in the images. An alternative approach is to use a large number or all of the voxels in the image. ${ }^{15}$ Voxel property methods are based on the assumption that some arithmetic combination of voxel values in two images, when applied to each image voxel alternately, gives a similarity measure that has an optimal value when the images are aligned. Although these techniques are widely used, the registration performance is limited in low-resolution imaging.

The registration of multimodal images can be non-image based if the imaging coordinate systems of the two devices involved are calibrated to each other. This requires the scanners to be in the same location and the assumption that the patient remains motionless between studies. ${ }^{10}$ This concept is the basis for the development of hybrid systems capable of performing both functional and structural imaging. These new devices include patient scanners allowing PET or SPECT and $\mathrm{CT}^{16-19}$ and small animal scanning combining PET and MRI. ${ }^{20}$

\section{INTEGRATED MODALITY IMAGING SYSTEMS: SPECT/CT AND PET/CT}

Hybrid systems provide functional and anatomical images in the same scanning session, and therefore limit the drawbacks of the other methods of image registration. ${ }^{21,22}$ Dual-modality imaging allows the two images to be acquired with consistent geometry and body habitus, with minimal delay between the two studies, and without the need for fiducial markers or complicated algorithms. Also the production of fused images is facilitated.

The design of a dual-modality device includes a radionuclide detector (SPECT or PET) and a CT scanner (x-ray tube and detector) mounted on a gantry with a patient table for nuclear medicine and x-ray imaging. ${ }^{23}$ The clinical applications of this new technology seem particularly useful in the field of oncology. Currently, only one commercial system with SPECT/CT capability is widely available. This hybrid imaging system combines a dual-detector, variable angle gamma camera with a low-dose $\mathrm{x}$-ray tube that is attached to the same rotating gantry as the gamma camera. This device provides, together with SPECT data, cross-sectional $\mathrm{x}$-ray transmission images that accurately locate the anatomical sites of radiotracer accumulations. Moreover, the gamma camera is capable of FDG imaging using coincidence detection. ${ }^{24,25}$

For acquiring the transmission data, the $\mathrm{x}$-ray tube and linear detector array rotate together around the patient in a fixed geometry, with a single slice imaged in 14 seconds. Multiple transmission slices are obtained by moving the table; the full field of view consists of 40 slices with the slice thickness fixed at $1 \mathrm{~cm}$. Radiation doses, calculated on the basis of phantom studies, range from $1.3 \mathrm{mGy}$ at the center to $5 \mathrm{mGy}$ at the surface. ${ }^{16}$ At the completion of the first type of acquisition (transmission or emission), the patient is automatically repositioned so that the $40-\mathrm{cm}$ axial field that was just scanned matches the 40$\mathrm{cm}$ axial field of view of the second imaging modality. Images are reconstructed in the workstation and transmission data are integrated in the nuclear medicine database; the alignment of slices is automatically performed, then matching pairs of x-ray and scintigraphic images are fused generating new images overlying SPECT and CT data. $^{26}$

Different types of dedicated PET/CT scanners are now commercially available. They incorporate high performance PET combined with multislice CT with 2-16 slices. ${ }^{27}$ Standard patient preparation used for PET and CT studies is applied to the PET/CT technology. After the patient 
is positioned on the scanner, an initial tomogram is performed to define the examination range for the PET/CT image acquisition. Then the spiral CT scan is performed. After CT acquisition, the scanner bed is moved to start the emission scan, whose duration and bed position varies with the detector technology used. ${ }^{28} \mathrm{CT}$ data are reconstructed to obtain images with the same slice thickness as the PET images to allow co-registration, then are transferred into the PET software environment. The reconstruction of emission images can be started while the PET scan of consecutive body areas is ongoing. ${ }^{29}$ Various methods for integrated visualization of the fused images are available in the different systems.

Besides acquiring co-registered functional and anatomical images, the first advantage of these hybrid systems is that the PET emission data can be corrected for photon-attenuation effects using the $\mathrm{CT}$ images. The total radiation burden for the CT of a PET/CT whole-body scan with a mA-s product of 40 (sufficient for anatomic localization) has been estimated to be $2-8 \mathrm{mSv}{ }^{30}$

\section{HYBRID SPECT/CT IN ONCOLOGY}

We recently evaluated the usefulness of the hybrid SPECT/CT system in consecutive patients. The value of fused SPECT/CT images was compared to SPECT alone. ${ }^{31}$ To this end, SPECT was first interpreted alone and then re-evaluated with the addition of fused images. SPECT/CT had a significant impact on the results in $41 \%$ of the patients, providing the correct localization of SPECT findings (Figs. 1 and 2), allowing definition of the functional significance of CT lesions and exclusion of disease in sites of physiologic tracer uptake. The fusion procedure was not time consuming and could be routinely employed to facilitate the interpretation of SPECT images.

Even-Sapir et al. ${ }^{32}$ evaluated the usefulness of the SPECT/CT device in patients with known or suspected endocrine tumors. Fused images improved imaging interpretation in patients with abnormal SPECT findings and provided information of clinical value, allowing more precise staging and prognosis, and better management of patients.

The benefit of combined SPECT/CT was prospectively assessed by Pfannenberg et al. ${ }^{33}$ in patients with neuroendocrine neoplasms. In this study, SPECT/CT accuracy was higher than that of SPECT alone; moreover, the results of imaged fusion altered treatment options in $28 \%$ of patients by excluding tumour lesions, sparing unnecessary surgery because of additional lesion detection, and changing the surgical approach or medical and radiopeptide therapy.

A recent study has evaluated the role of the hybrid gamma camera/low-dose CT device in lymphoscintigraphy for sentinel node mapping. ${ }^{34} \mathrm{~Pa}-$ tients with malignant cutaneous melanoma and squamous cell carcinoma scheduled for sentinel node biopsy were considered. SPECT/CT imaging identified sentinel lymph-nodes missed on conventional lymphoscintigraphy. Moreover, fused images added clinically relevant data in $50 \%$ of patients with trunk melanoma, $44 \%$ of patients with head and neck cancer, and none in patients with limb melanoma. These results indicated that the precise anatomical localization of sentinel nodes obtained by SPECT/CT imaging was useful for a better surgery planning in patients with head and neck or trunk melanoma and in patients with head and neck cancer.

Hybrid imaging in lymphoma patients studied with Ga-67 scintigraphy gave additional diagnostic information in $40 \%$ of patients, distinguishing pathological from physiological uptake and leading to the diagnosis of previously undetected sites of disease. ${ }^{35}$ When used in monitoring response to treatment and follow-up of lymphoma patients, SPECT/CT provided better localization of lesions visible on Ga-67 SPECT, adding clinically useful information in $50 \%$ of cases by showing the relationship between residual mass and residual tumor, guiding biopsy or indicating the correct radiotherapy planning. ${ }^{36}$

The role of hybrid imaging in evaluating oncological patients with suspected bone metastases was assessed by Eschmann et al. ${ }^{37}$ in a series of patients with equivocal findings on routine bone scintigraphy with Tc-99m diphosphonate. SPECT/CT allowed a correct classification of $81 \%$ of indeterminate lesions, in particular identifying the benign lesions and so improving the specificity of the bone scan.

Further applications of SPECT/CT in oncology may include the use of monoclonal antibodies both for diagnosis ${ }^{38}$ and dosimetric estimations for target radionuclide therapy. ${ }^{39}$

\section{HYBRID PET/CT IN ONCOLOGY}

The first clinical studies evaluating the advantages of PET/CT imaging over PET scan alone 

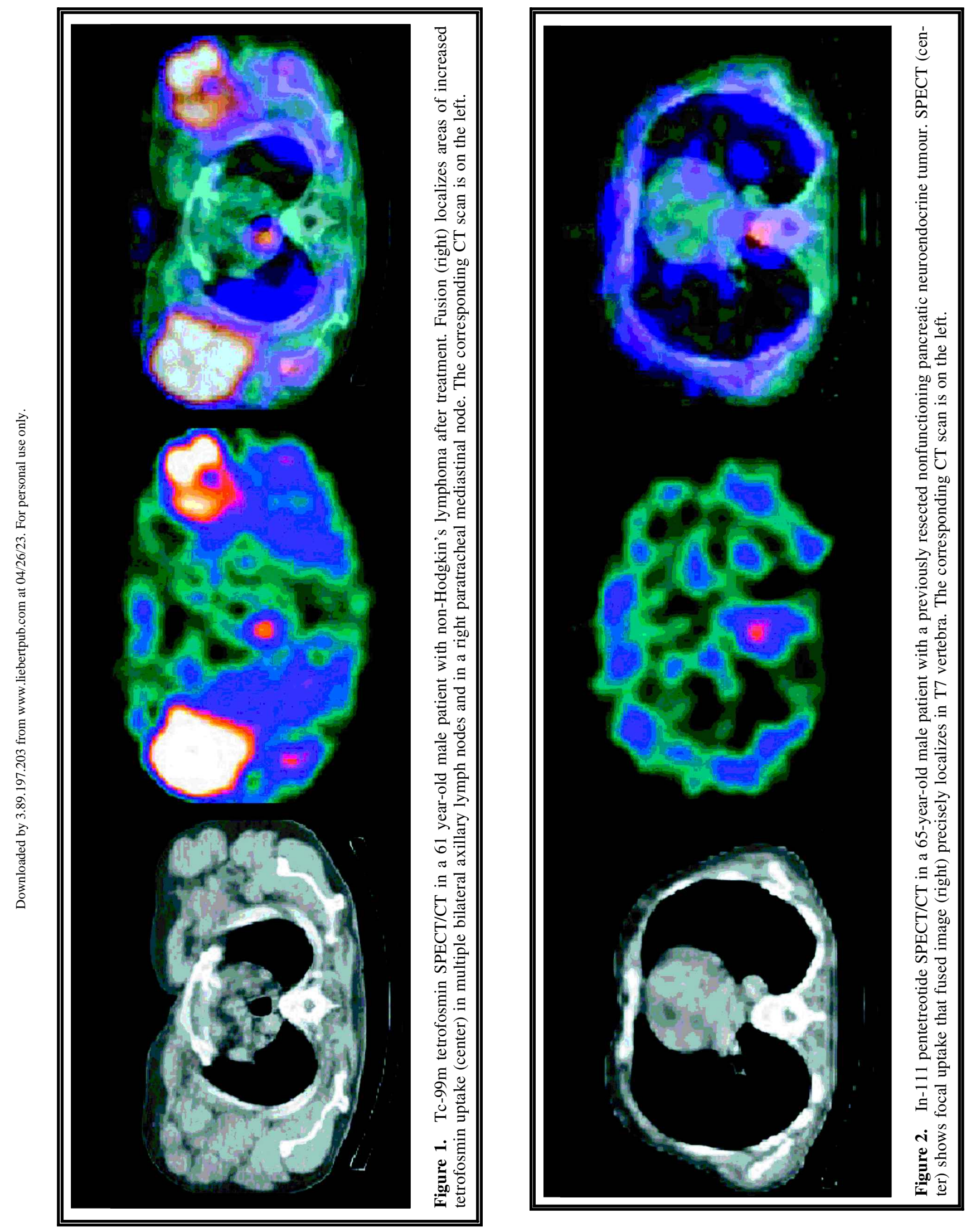
were performed at The University of Pittsburgh, Pittsburgh, PA, using a prototype PET/CT scanner. Martinelli et al. ${ }^{40}$ reviewed more than 100 studies in patients with a variety of malignancies. PET/CT offered significant advantages including more accurate localization of FDG-uptake, distinction of pathological from physiologic uptake, and improvements in monitoring treatment. The same group also investigated the impact of PET/CT in a small series ${ }^{41}$ : hybrid imaging positively affected patient management through localization for surgical and radiation therapy planning as well as assessment of tumor response.

The initial applications of PET/CT for tumor staging was investigated by Hany et al. ${ }^{42}$ On a patient-by-patient analysis, PET alone allowed correct staging in $72 \%$ of the patients, whereas PET/CT enabled correct staging in $92 \%$ of the patients. On a lesion-by-lesion analysis, accuracy for detecting tumor sites was $91 \%$ for PET alone and $98 \%$ for PET/CT. False positive cases were reduced by PET/CT due to identification of normal physiologic variants of FDG accumulation. False negative results were reduced by correct localization of tumor lesions (Fig. 3).

Bar-Shalom et al. ${ }^{43}$ have recently assessed the clinical performance of PET/CT in 204 oncological patients. Their data show that hybrid imaging has an impact on both diagnostic and therapeutic aspects of patient management. PET/CT improved the diagnostic interpretation of PET and CT in $49 \%$ of patients and $30 \%$ of sites. Fused images allowed more precise lesion characterisation and localization, and retrospective lesion detection both in different tumors and stages of disease. Moreover, PET/CT had an impact on patient management in $14 \%$ of cases, assisting in planning therapy, obviating the need for further evaluation, and guiding further diagnostic procedures.

The new technology of PET/CT seems particularly useful in evaluating patients with head and neck tumors. ${ }^{44}$ In this area, various normal structures demonstrate variable FDG uptake. ${ }^{45}$ The incremental diagnostic value of PET/CT fusion imaging in patients with head and neck cancers was investigated by Schoder et al. ${ }^{46}$ In this study, PET/CT detected additional tumors and significantly decreased the number of equivocal findings of PET alone.

In non-small-cell lung cancer, PET/CT proved superior to PET alone, CT alone, and visually correlated PET and CT in staging the disease. ${ }^{47}$ In particular, tumor staging was significantly more accurate. Nodal staging was more accurate because PET/CT imaging clearly identified hilar, mediastinal, and supraclavicular lymph-nodes (Fig. 4). However, with PET/CT, spatial misregistration of primary lung lesions ${ }^{48}$ and respiratory motion artefacts ${ }^{49}$ may occur.

A recent study comparing FDG PET and PET/CT in 45 patients with colorectal cancer demonstrated that PET/CT imaging increased the accuracy and certainty of locating lesions in this neoplasm. ${ }^{50}$ PET/CT reduced the frequency of equivocal and probable lesion by $50 \%$, increased the frequency of definite lesion characterization by $30 \%$ and the number of definite locations by $25 \%$. These results provided an improvement in staging and restaging accuracy from $78 \%$ to $89 \%$ in a patient-by-patient analysis.

A preliminary report by Makhija et al. ${ }^{51}$ suggested that PET/CT can be useful for identifying patients with recurrent ovarian and fallopian tube carcinoma. These data have been recently confirmed in a series of patients with ovarian cancer, rising serum CA125 levels, and negative or equivocal conventional CT imaging after primary therapy. PET/CT images demonstrated high sensitivity and positive predictive value in identifying potentially resectable, macroscopic recurrent ovarian cancer. ${ }^{52}$

The role of PET/CT in radiotherapy treatment planning was prospectively evaluated by Ciernik et al. ${ }^{53}$ in patients with solid tumors scheduled to undergo external beam radiation. Combined $\mathrm{PET} / \mathrm{CT}$ changed the radiation treatment strategy from curative to palliative in $16 \%$ of the patients due to the detection of distant metastases. Moreover, the target volume was changed in $46 \%$ of cases. These results indicate that the use of PET/CT for treatment planning for radiation therapy improves the standardization of volume delineation compared with that of $\mathrm{CT}$ alone. PET/CT can reduce the risk for geographic misses and minimize the dose of ionizing radiation applied to non-target organs. However, the impact of these modifications on patient outcomes will need to be addressed.

A number of other clinical applications of PET/CT are currently under investigation ${ }^{44}$; future work may be in tumors with an unknown primary, in bone and soft-tissue malignancies, and in lymphoma.

\section{CONCLUSION}

Imaging fusion is the process that allows the integration of the functional information of nuclear medicine studies with the anatomic strengths of 


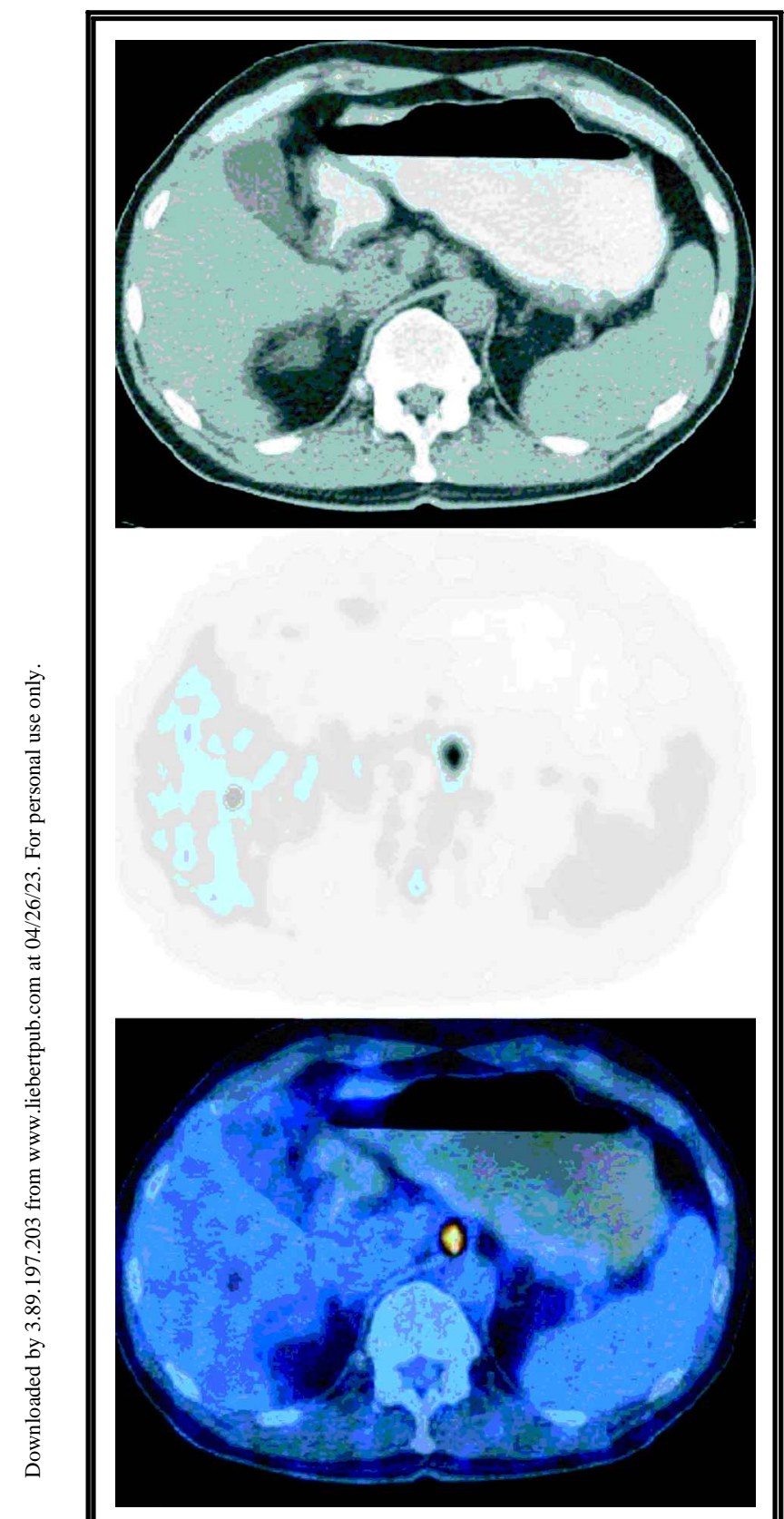

Figure 3. FDG PET/CT in a 72-year-old male patient with gastric cancer after chemotherapy. PET alone (middle) depicts focal FDG uptake that PET/CT (bottom) precisely localizes in a single paragastric lymph node between the aorta and the stomach. The corresponding CT scan is at the top. (Image courtesy of Thomas F. Hany, M.D., and Gustav K. von Schulthess, M.D., Ph.D., Institute of Nuclear Medicine, University Hospital Zurich, Zurich, Switzerland.)

structural modalities, providing the solution to a variety of clinical problems, especially in cancer patients. SPECT/CT and PET/CT are recently developed dual-modality, combined functional/ morphologic imaging devices. They are able to

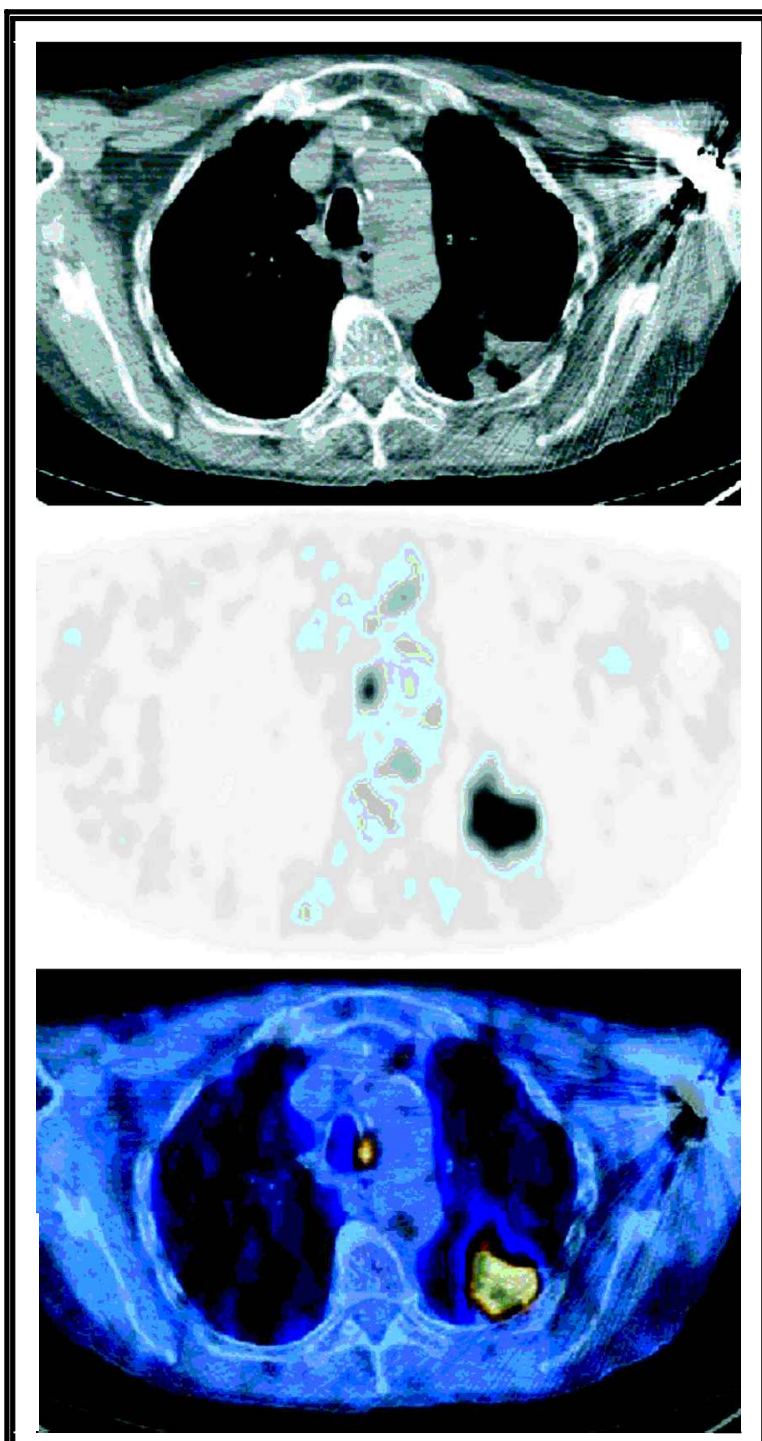

Figure 4. FDG PET/CT in a 67-year-old male patient with non-small-cell left lung cancer. PET alone (middle) shows FDG uptake in the left hemithorax and in the mediastinum. PET/CT (bottom) reveals that these activities are located in the left apical lower lobe segment and in an ipsilateral, peribronchialmediastinallymph node. The corresponding CT scan is at the top. (Image courtesy of Thomas F. Hany, M.D., and Gustav K. von Schulthess, M.D., Ph.D., Institute of Nuclear Medicine, University Hospital Zurich, Zurich, Switzerland.)

overcome some drawbacks of conventional image fusion approaches and are routinely utilizable. The data available in oncological applications clearly indicate that these technologies improve scintigraphic imaging interpretation and affect clinical strategies. In the future, hybrid imaging will play an increasing role in cancer diagnosis, staging, monitoring, and treatment planning, so improving patient management. 


\section{ACKNOWLEDGMENTS}

The authors thank Dr. Thomas F. Hany and Prof. Gustav K. von Schulthess from the Institute of Nuclear Medicine, University Hospital Zurich, Switzerland, for kindly supplying the PET/CT images, and Drs. Roberta Danieli, Carlo Manni, Valentina Picardi, Francesca Capoccetti, and Ana Maria Samanes Gajate for their assistance.

\section{REFERENCES}

1. Treves ST, Mitchell KD, Habboush IH. Three-dimensional image alignment, registration and fusion. $\mathrm{J} \mathrm{Nucl}$ Med 1998;42:83.

2. Coreia JA. Registration of nuclear medicine images. $J$ Nucl Med 1990;31:1227.

3. Weber DA, Ivanovic M. Correlative image registration. Semin Nucl Med 1994;24:311.

4. Cook JR, Ott RJ. Dual-modality imaging. Eur Radiol 2001;11:1857.

5. Israel O, Keidar Z, Iosilevsky G, et al. The fusion of anatomic and physiologic imaging in the management of patients with cancer. Semin Nucl Med 2001;31:191.

6. Shreve PD. Adding structure to function. $\mathrm{J} \mathrm{Nucl} \mathrm{Med}$ 2000;41:1380.

7. Wagner HN. Images of the future. J Nucl Med 1978;19: 599.

8. Maisey MN, Hawkes DJ, Lukswiecki-Vydelingum AM. Synergistic imaging. Eur J Nucl Med 1992;19:1002.

9. Kramer EL, Noz ME. CT-SPECT fusion for analysis of radiolabeled antibodies: Applications in gastrointestinal and lung carcinoma. Nucl Med Biol 1991;18:27.

10. Maintz JB, Viergever MA. A survey of medical imaging registration. Med Image Anal 1998;2:1.

11. Hutton BF, Braun M, Thurfjell L, Lau DYH. Image registration: An essential tool for nuclear medicine. Eur $J$ Nucl Med Mol Imaging 2002;29:559.

12. Hill DLG, Hawkes DJ, Crossman JE, et al. Registration of MR and CT images for skull base surgery using point-like anatomical features. Br J Radiol 1991;64: 1030 .

13. Thirion J. New feature points based on geometric invariants for 3D image registration. Int J Comp Vision 1996;18:121.

14. Pellizzari CA, Chen GTY, Spelbring DR, et al. Accurate three-dimensional registration of CT, PET and/or MR images of the brain. J Comput Assist Tomogr 1989;13:20.

15. Hutton BF, Braun M. Software for image registration: Algorithms, accuracy, efficacy. Semin Nucl Med 2003; 33:180.

16. Bocher M, Balan A, Krausz Y, et al. Gamma cameramounted anatomical x-ray tomography: Technology, system characteristics and first images. Eur J Nucl Med 2000;27:619.

17. Patton JA, Delbeke D, Sandler MP. Image fusion using an integrated-dual-head coincidence camera with x-ray tube-based attenuation maps. J Nucl Med 2000;41:1364.

18. Beyer T, Townsend D, Brun T, et al. A combined PET/CT scanner for clinical oncology. J Nucl Med 2000;41:1369.

19. Townsend, D Beyer T. A combined PET/CT scanner: The path to true image fusion. Br J Radiol 2002;75:S24.

20. Shao Y, Cherry SR, Farahani K, et al. Development of a PET detector system compatible with MRI/NMR systems. IEEE Trans Nucl Sci 1997;NS-44:1167.

21. von Schulthess GK, Pelc NJ. Integrated-modalityimaging: The best of both words. Acad Radiol 2002;9:1241.

22. Townsend DW, Cherry SR. Combining anatomy with function: The path to true image fusion. Eur Radiol 2001;11:1968.

23. Hasegawa BH, Iwata K, Wong KH, et al. Dual-modality imaging of function and physiology. Acad Radiol 2002;9:1305.

24. Delbeke D, Martin WH, Patton JA et al. Value of iterative reconstruction, attenuation correction, and image fusion in the interpretation of FDG PET images with an integrated dual-head coincidence camera and x-ray based attenuation maps. Radiology 2001;218:163.

25. Israel O, Mor M, Gaitini D, et al. Combined functional and structural evaluation of cancer patients with a hybrid camera-based PET/CT system using ${ }^{18}$ F-FDG. $J$ Nucl Med 2002;43:1129.

26. Schillaci O, Danieli R, Picardi V, et al. A combined SPET/CT imaging system for functional anatomical mapping: initial clinical applications. Eur J Nucl Med Mol Imaging 2002;29 (Suppl. 1):S94.

27. Townsend DW, Beyer T, Blodgett, TM. PET/CT scanners: A hardware approach to image fusion. Semin $\mathrm{Nucl}$ Med 2003;33:193.

28. Costa DC, Visvikis D, Crosdale I, et al. Positron emission and computed x-ray tomography: A coming together. Nucl Med Commun 2003;24:351.

29. Hany TF, Berthold T. PET/CT imaging protocol. In: von Schulthess GK, ed. Clinical Molecular Anatomic Imaging. Philadelphia: Lippincott Williams \& Wilkins; 2003:141.

30. Kneifel S. Radiation dose and radiation protection. In: von Schulthess GK, ed. Clinical Molecular Anatomic Imaging. Philadelphia: Lippincott Williams \& Wilkins; 2003:68.

31. Schillaci O, Danieli R, Manni C, et al. The added clinical value of SPET/CT with a hybrid imaging system. J Nucl Med 2003;44:290P.

32. Even-Sapir E, Keidar Z, Sachs J, et al. The new technology of combined transmission and emission tomography in evaluation of endocrine neoplasms. J Nucl Med 2001;42:998.

33. Pfannenberg AC, Eschmann SM, Horger M, et al. Benefit of anatomical-functional image fusion in the diagnostic work-up of neuroendocrine neoplasms. Eur $J$ Nucl Med Mol Imaging 2003;30:835.

34. Even-Sapir E, Lerman H, Lievshitz G, et al. Lymphoscintigraphy for sentinel node mapping using a hybrid SPECT/CT system. J Nucl Med 2003;44:1413.

35. Israel O, Yefremov N, Mor M, et al. A new technology 
of combined transmission (CT) and emission (Ga-67) tomography (TET) in the evaluation of patients with lymphoma [abstr]. J Nucl Med 2000;41:70P.

36. Israel O, Yefremov N, Mor M, et al. Combined transmission Ga-67 emission tomography (TET) in the evaluation of response to treatment and diagnosis of recurrence in patients with lymphoma. Eur J Nucl Med 2000;27:1160.

37. Eschmann SM, Horger M, Pfannenberg AC, et al. Improved specificity of bone scintigraphy by combined transmission and emission tomography (TET). J Nucl Med 2002;43:340P.

38. Carrol MJ, Britton KE. Hawkeye mediated multiple image fusion applied to prostate cancer detection. Eur $J$ Nucl Med Mol Imaging 2002;29 (supple. 1):S94.

39. Koral KF, Dewaraja Y, Li J, et al. Update on hybrid conjugate-view SPECT tumor dosimetry and response in 131I-tositumomab therapy of previously untreated lymphoma patients. J Nucl Med 2003;44:457.

40. Martinelli M, Townsend D, Meltzer C, Villemagne VV. Survey of results of whole body imaging using PET/CT at the University of Pittsburgh Medical Center PET Facility. Clin Positron Imaging 2000;3:161.

41. Kluetz, PG, Meltzer CC, Villemagne VL, et al. Combined PET/CT imaging in oncology: Impact on patient management. Clin Positron Imaging 2000;3:223.

42. Hany TF, Steinert HC, Goerres GW, et al. PET diagnostic accuracy: improvement with in-line PET-CT systems: initial results. Radiology 2002;225:575.

43. Bar-Shalom R, Yefremov N, Guralnik L, et al. Clinical performance of PET/CT in evaluation of cancer: additional value for diagnostic imaging and patient management. J Nucl Med 2003;44:1200.

44. Schoder H, Erdi E, Larson SM, Yeung HWD. PET/CT: A new imaging technology in nuclear medicine. Eur $J$ Nucl Med Mol Imaging 2003;30:1419.
45. Goerres GW, Von Schulthess GK, Hany TF. Positron emission tomography and PET CT of the head and neck: FDG uptake in normal anatomy, in benign lesions, and in changes resulting from treatment. AJR Am J Roentgenol 2002;179:1337.

46. Schoder H, Yeung H, Larson SM. Incremental diagnostic value of PET/CT fusion imaging in patients with head and neck malignancies. Radiology 2002;225(P): 333.

47. Lardinois D, Weder W, Hany TF, et al. Staging of nonsmall-cell lung cancer with integrated positron-emission tomography and computed tomography. $N$ Engl J Med 2003;348:2500.

48. Cohade C, Osman M, Marshall LN, Wahl RL. PET-CT: Accuracy of PET and CT spatial registration of lung lesions. Eur J Nucl Med Mol Imaging 2003;30:721.

49. Osman MM, Cohade C, Nakamoto Y, Wahl RL. Respiratory motion artifacts on PET emission images obtained using CT attenuation correction on PET-CT. Eur J Nucl Med Mol Imaging 2003;30:603.

50. Cohade C, Osman M, Leal J, Wahl RL. Direct comparison of (18)F-FDG PET and PET/CT in patients with colorectal carcinoma. J Nucl Med 2003;44:1797.

51. Makhija S, Howden N, Edwards R, et al. Positron emission computed tomography/computed tomography imaging for the detection of recurrent ovarian and fallopian tube carcinoma: A retrospective review. Gynecol Oncol 2002;85:53.

52. Bristow RE, del Carmen MG, Pannu HK, et al. Clinically occult recurrent ovarian cancer: Patient selection for secondary cytoreductive surgery using combined PET/CT. Gynecol Oncol 2003;90:519.

53. Ciernik IF, Dizendorf E, Baumert BG, et al. Radiation treatment planning with an integrated positron emission and computer tomography (PET/CT): A feasibility study. Int J Radiat Oncol Biol Phys 2003;57:853. 


\section{About the Authors}

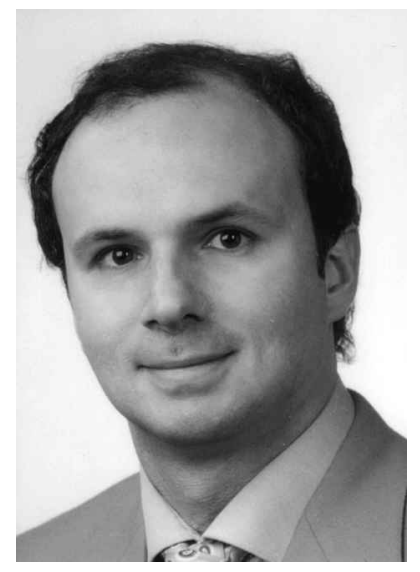

Orazio Schillaci received his medical education at the University of Rome "La Sapienza," Rome, Italy, where he also obtained his Ph.D. in functional radioisotope imaging. In 1999, he was a visiting professor at the University of California Davis, Sacramento, $C A$, in the Section of Radiodiagnosis and Therapy. He is currently an associate professor of nuclear medicine in the department of biopathology and diagnostic imaging, at the University of Rome "Tor Vergata," Rome, Italy, and chief of the Section of Nuclear Medicine at the Policlinico Tor Vergata of Rome, Rome, Italy.

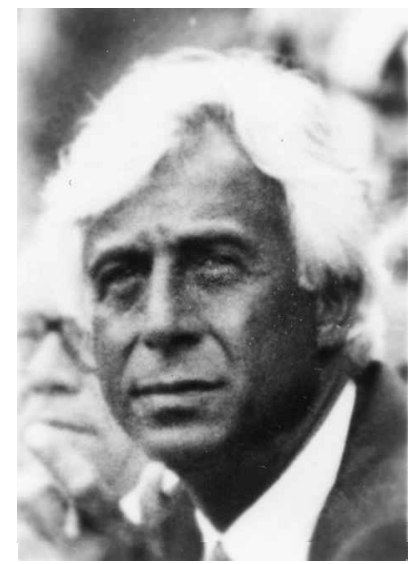

Giovanni Simonetti received his medical degree from the University of Sassari. He is a full Professor of Radiology and of Biopathology and Diagnostic Imaging at the University of Rome "Tor Vergata," Rome, Italy, and the chief of the Department of Diagnostic Imaging and Interventional Radiology at the Policlinico Tor Vergata of Rome, Rome, Italy. He is currently the director of both the Specialty Schools in Radiodiagnosis and Radiotherapy at the University of Rome "Tor Vergata," and the president of the Senology Task Group of the Italian Society of Medical Radiology. 